\title{
La cirugía bariátrica se asocia con una mayor sobrevida a largo plazo en pacientes con obesidad mórbida
}

\author{
Bariatric surgery is associated with increased long-term survival in morbidly obese patients
}

Arterburn D et al. JAMA 2015;313(1):62-70

\section{Objetivos}

Evaluar la sobrevida a largo plazo en una cohorte de veteranos estadounidenses que se realizaron cirugía bariátrica (CB) en comparación con un grupo control.

\section{Diseño, lugar y pacientes}

Estudio de cohorte retrospectivo. Realizado en Durham, Pittsburgh. Estados Unidos.

Se seleccionaron 2752 pacientes con CB realizada en los distintos centros bariátricos de veteranos, entre enero del 2000 y septiembre del 2011. Se excluyeron 252 pacientes, por pérdida en sus registros de indice de masa corporal (IMC), CB no estandarizada, IMC $<35$, condiciones médicas y características no compatibles para el control.

Se comparó la cohorte de 2500 pacientes con 7462 controles de características y comorbilidades similares (edad, sexo, estado civil, IMC, costos hospitalarios, hipertensión, dislipemia, artritis, depresión, enfermedad coronaria, reflujo gastroesofági- co, asma, hígado graso, síndrome de ovario poliquístico, abuso de alcohol y sustancias, esquizofrenia).

\section{Evaluación de factores pronósticos}

Se evaluó la mortalidad a uno, cinco y diez años. Se realizó estimación de Kaplan-Meier y el modelo de regresión de Cox, para el análisis multivariado. Se comparó un subgrupo de pacientes acorde al sexo, la evolución de su diabetes, la superobesidad (IMC >50) y el periodo de realización de la cirugía.

\section{Resultados principales}

Se registraron 263 muertes en el grupo quirúrgico (promedio de 52 años e IMC 47) tras una media de seguimiento de 6,9 años y 1277 en el grupo control (53 años e IMC 46) seguido durante 6,6 años. El análisis multivariado se muestra en la tabla 1. No se encontraron diferencias significativas entre subgrupos por sexo, diabetes y superobesidad.

Tabla 1. Asociación entre cirugía bariátrica y sobrevida.

\begin{tabular}{c|c|c|c|c|c|c|c}
\multirow{2}{*}{ Intervalo } & \multicolumn{2}{|c|}{ Grupo quirúrgico } & \multicolumn{2}{|c|}{ Grupo control } & \multicolumn{2}{c|}{ Efecto (IC 95\%) } & \multirow{2}{*}{ P } \\
\cline { 2 - 7 } & $\mathbf{N}$ & Óbitos & N & Óbitos & Hazard Ratio & NNT & \\
\hline Hasta 1 año & 2500 & 61 & 7462 & 129 & $1,28(0,98 \mathrm{a} 1,68)$ & - & 0,07 \\
\hline 1 a 5 años & 2439 & 86 & 7266 & 554 & $0,45(0,36 \mathrm{a} 0,56)$ & $24(20 \mathrm{a} 32)$ & $<0,001$ \\
\hline 5 a 14 años & 1657 & 116 & 4624 & 594 & $0,47(0,39 \mathrm{a} 0,58)$ & $17(14 \mathrm{a} 23)$ & $<0,001$ \\
\hline
\end{tabular}

NNT: número necesario a tratar. IC 95\%: intervalo de confianza del 95\%.

\section{Conclusiones}

La cirugía bariátrica se asoció con una mejor sobrevida a largo plazo.
Fuente de financiamiento/conflictos de interés: Health Services Research and Development (Department of Veterans Affairs)/ Uno de los autores (Dr Eid) declaró recibir honorarios personales de Apollo Endosurgery, Bariatric Fusion, Cooper Surgical, and Covidie.

\section{Comentario}

La obesidad es un problema sanitario muy importante, con un aumento sostenido de la prevalencia en los últimos años. Se calcula que el sobrepeso y la obesidad causan 3,4 millones de muertes por año en el mundo. El porcentaje de los pacientes con un IMC >25 se incrementó de $28,8 \%$ en 1980 , a 36,9\% en 2013 en hombres y fue similar en la población femenina'. La mayoría de los estudios epidemiológicos han mostrado un aumento de la mortalidad en pacientes obesos, con una disminución de la expectativa de vida de entre 5 y 20 años. Múltiples publicaciones muestran la efectividad de la $\mathrm{CB}$ con respecto al descenso de peso y la mejoría o resolución de comorbilidades como hipertensión arterial y diabetes tipo 2 en el corto y mediano plazo ( 2 a 5 años). ${ }^{2}$ Hay pocos estudios que evalúan los resultados de la $\mathrm{CB}$ con respecto a la sobrevida. EI Swedish Obesity Subjects Trial, cuyo primer objetivo era evaluar la mortalidad global de la CB, comparó 2010 pacientes obesos operados vs. 2037 pacientes control; recolectó información en forma prospectiva desde finales de la década del 80 y mostró una reducción en la mortalidad de casi el $30 \%$ tras un seguimiento de más de 16 años. ${ }^{3}$ El presente trabajo presenta un análisis retrospectivo de cohorte, comparando pacientes obesos mórbidos operados (bypass gástrico, manga gástrica o banda gástrica principalmente) con pacientes obesos mórbidos apareados por similares características. Luego de 14 años de duración del estudio, se observó una disminución significativa en la mortalidad global e impactantes número necesario a tratar, siendo dichos resultados consistentes en diferentes subgrupos definidos por sexo, diabetes y superobesidad. Una limitación importante radica en el diseño del mismo, ya que es un estudio observacional retrospectivo donde se analizaron varias técnicas quirúrgicas. De todas formas, sus resultados cobran relevancia considerando que en la actualidad no hay estudios aleatorizados que evalúen sobrevida a largo plazo en CB.

\section{Conclusiones del comentador}

Actualmente la $\mathrm{CB}$ es un procedimiento seguro conducido por equipos multidisciplinarios, con bajas tasas de morbimortalidad, con beneficios claros en cuanto al descenso de peso, tratamiento de comorbilidades y mejoría en la calidad de vida. La realización de estos procedimientos por vía mini-invasiva (laparoscópica) y los resultados favorables incluso a largo plazo, han contribuido a la expansión del número de procedimientos realizados por año. Más investigaciones son necesarias para determinar el impacto en la sobrevida de la CB en diferentes grupos de pacientes.

Fernando Gabriel Wright [ Servicio Cirugía General. Sección Cirugía Bariátrica. Hospital Italiano de Buenos Aires]

Juan Rodolfo Medici [ Servicio Cirugía General. Sección Cirugía Bariátrica. Hospital Italiano de Buenos Aires]

Wright FG, Medici JR. La cirugía bariátrica se asocia con una mayor sobrevida a largo plazo en pacientes con obesidad mórbida. Evid Act Pract Ambul $2016 ; 19(4): 117$. Comentado de: Arterburn DE, y col. Association between bariatric surgery and long-term survival. JAMA. 2015;313(1):62-70. PMID: 25562267.

Referencias:

1. Ng M, Fleming T, Robinson M, Thomson B, y col. Global, regional, and national prevalence of overweight and obesity in children and adults during 1980-2013: a systematic analysis for the Global Burden of Disease Study 2013. Lancet. 2014;384(9945):766.

2. Singh AK, Singh R, Kota SK. Bariatric surgery and diabetes remission: Who would have thought it? Indian J Endocrinol Metab. 2015; 19(5): 563-576.

3. Sjöström L. Review of the key results from the Swedish Obese Subjects (SOS) trial—a prospective controlled intervention study of bariatric surgery. J Intem Med. 2013;273(3):219-234 